\title{
Strongyloidiasis in Po valley: serological and parasitological screening in blood donors of Cremona
}

\author{
Paolo Mangoni, Lucio Ferrari, Giuliana Mazzei, Francesco Bernieri \\ Struttura Semplice di Microbiologia, A.O. "Istituti Ospitalieri di Cremona", Cremona
}

Key words: Strongyloides stercoralis, Strongyloidiasis, Iperinfestation syndrome, Parasite, Cremona, Blood donors

\section{Strongiloidosi in Pianura Padana: screening sierologico e parassitologico in donatori di sangue cremonesi}

\section{SUMMARY}

Backgroud. Strongyloides stercoralis is a ubiquitous nematode that is present also in Italy in a hypoendemic form, especially in the Po Valley.

It is responsable for strongyloidiasis, an intestinal parasitosis usually asymptomatic or paucy-symptomatic that occasionally can cause a hyperinfestation syndrome and disseminated disease.

Objectives. To determine the presence of $S$. stercoralis in asympomatic people afferent to the Cremona hospital by serological screening and direct microscopic search.

Study Design. In the period from December I, 2010 to June 30, 201 I we screened I54 donors with absolute eosinophilia $>500 / \mu \mathrm{L}$ and percentage of eosinophils $\geq 9 \%$.

The research of anti-S. stercoralis antibodies was performed through an ELISA method (S.ratti Bordier Products). Donors positive to the screening test were tested for direct parasite search through with phormol-ethyl acetate stool concentration (FEA) and culture on S. stercoralis Agar (Biolife), Milan).

Results. 13 donors were positive to the screening test $(8.4 \%)$. 10 of these 13 donors performed coproparassitologic examination. S. stercoralis was identified in the stools of $3 / 10$ tested patients through culture and in 2/10 through FEA.

Conclusions. The study results are in line with the data reported in the literature for similar geographic areas. Among the risk factors are to be counted the type of job (agriculture), and the domicile in the rural environment. The culture in S. stercoralis Agar has proved much more sensitive than FEA.The serological diagnosis represent a valuable contribution to traditional coproparassitologic examination, particularlywhen screening asymptomatic individuals at risk.

\section{INTRODUZIONE}

Strongyloides stercoralis, nematode appartenente alla famiglia Strongyloididae è responsabile della strongiloidosi, una parassitosi intestinale con decorso solitamente asintomatico o paucisintomatico che si può persistere per anni grazie all'autoinfestazione e che, occasionalmente, a seguito di condizioni favorenti, può divenire ingravescente causando la sindrome da iperinfestazione (SI) e la strongiloidosi disseminata (SD) (4).

L'infestazione si acquisisce tramite la penetrazione attiva attraverso la cute integra delle larve filariformi (L3) presenti nel suolo.

$\mathrm{Ne}$ consegue che i maggiori fattori di rischio per acquisire l'infestazione da $S$. stercoralis sono lavori a contatto con terreno come l'agricoltura e il giardinaggio.

Occasionalmente, come dimostrato per $S$. $f$. fülleborni e $S$. f. kellyi, le larve possono essere trasmesse anche attraverso il latte materno.

Non sembra esclusa anche una trasmissione ani- male-uomo soprattutto in riferimento ad animali domestici come cani e gatti $(2,4,5,8)$.

S. stercoralis è in grado di causare autoinfestazione. Si ha autoinfestazione esterna quando larve rabditoidi maturano a filarioidi nei residui fecali presenti nell'area perianale con conseguente penetrazione attraverso la cute del perineo, interna quando le larve rabditoidi maturano a larve filarioidi nell'intestino prima di venire escrete con le feci, con conseguente penetrazione attraverso la parete intestinale.

Autoinfestazione interna, a basso livello, è sempre presente ed è responsabile del mantenimento della parassitosi; che nell'ospite parassitato con buone condizioni immunitarie permette l'instaurasi di un equilibrio pluriennale, anche di decine di anni.

In caso di immunodepressione, anche iatrogena per uso prolungato di corticosteroidi, l'infestazione può divenire ingravescente causando strongiloidosi maligna che si manifesta sotto forma di sindrome da iperinfestazione (SI) mortale in circa 
il $15 \%$ dei casi o di strongiloidosi disseminata (SD) mortale in circa $1^{\prime} 80 \%$ dei casi (4).

È stimata una popolazione infestata di circa 70 milioni di soggetti, la maggior parte dei quali residenti in zone della fascia tropicale e sub tropicale. Tuttavia $S$. stercoralis è considerato un nematode ubiquitario in quanto riscontrato anche in regioni temperate, zone ipoendemiche, tra le quali anche Paesi industrializzati come l'Italia (epicentro in Pianura Padana), Svizzera, sud della Francia, Spagna, Regno Unito, Grecia, Europa orientale (Polonia, Ungheria, Romania, Bulgaria), stati meridionali dell'ex U.R.S.S., zone a prevalente attività agricola nel sud degli U.S.A. (Kentucky, Tennessee), Giappone meridionale (isola di Ryukyu), Australia (popolazioni aborigene) $(3,6)$. Nelle zone temperate ipoendemiche l'infestazione è strettamente correlata alla attività lavorativa agricola e si ritiene che nella maggior parte dei casi l'età media dei soggetti autoctoni infestati sia elevata (circa 70-80 anni) e che l'acquisizione primaria dell'infestazione possa risalire anche a decenni precedenti l'eventuale diagnosi parassitologia.

L'infestazione era più frequente nel periodo compreso tra le due guerre mondiali, ma anche nel secondo dopoguerra fino almeno agli anni '60 dello scorso secolo, la popolazione rurale era molto numerosa e spesso in condizioni economiche disagiate, a volte prossime all'indigenza, e frequentemente viveva in condizioni igieniche carenti (abitazioni prive di latrine).

Oggi il miglioramento delle condizioni igieniche della vita quotidiana dei contadini e la diffusa meccanizzazione dell'attività agricola hanno ridotto i contatti delle persone con terra e fango, riducendo il numero di infestazioni nelle fasce di età ancora in attività lavorativa.

Nonostante la diminuzione delle infestazioni, studi recenti (4) hanno dimostrato la persistenza di $S$. stercoralis anche in paesi industrializzati e messo in evidenza l'importanza di ricercare tale nematode anche in queste realtà dove l'uso massivo di sostanze immunosoppressive e farmaci citotossici sono molto diffusi e potrebbero favorire l'insorgenza di complicanze molto severe quali SD e SI.

Al giorno d'oggi risulta quindi necessario identificare con sicurezza $i$ "portatori asintomatici" di tale parassita tra soggetti a rischio di poter sviluppare una iperinfestazione o un'infezione disseminata.

La letteratura non riporta molti dati di prevalenza della strongiloidosi nella nostra regione e soprattutto non sono molte le strutture che eseguono test diagnostici completi che comprendano anche la ricerca di anticorpi specifici.
Partendo dai risultati di alcuni studi $(1,3)$, abbiamo voluto indagare l'eventuale presenza di strongiloidosi non sintomatica cercando di determinarne la prevalenza tra i donatori di sangue afferenti all'A.O. Istituti Ospitalieri di Cremona, mediante uno screening sierologico preliminare completato da ricerca parassitologia diretta del parassita sui sieropositivi, al fine di conoscere la situazione nella nostra area geografica e poter sensibilizzare i medici di reparto e di medicina generale su tale problematica.

\section{MATERIALI E METODI}

Il periodo preso in considerazione nel nostro studio va dal 01 dicembre 2010 al 30 giugno 2011.

Valutando gli esiti degli esami ematochimici di 9076 donatori di sangue afferenti presso il centro trasfusionale dell'A.O. Istituti Ospitalieri di Cremona e sezioni AVIS limitrofe, sono stati selezionati 154 donatori che presentavano un numero assoluto di eosinofili (EOn) superiore a 500 cellule/ $\mu \mathrm{L}$ ed una percentuale di eosinofili (EC\%) pari o superiore al $9 \%$; sui sieri di questi donatori è stato effettuato uno screening sierologico specifico.

La ricerca di anticorpi anti-S. stercoralis è stata eseguita con il metodo ELISA $S$. ratti (Bordier Affinity Products, $\mathrm{CH}$, distribuito da Effegiemme srl, Milano), dotato di sensibilità e specificità dichiarate rispettivamente dell' 88 e del $95 \%(7,9)$. Il kit era costituito da 96 pozzetti sensibilizzati con antigeni larvali somatici di S. ratti.

Ogni donatore risultato positivo all'esame sierologico di screening è stato contattato per chiedere la disponibilità a sottoporsi ad un esame coproparassitologico seriato su 3 campioni di feci raccolti a giorni alterni, e conservati in frigorifero $\left(4^{\circ} \mathrm{C}\right)$ fino al momento della consegna.

Al momento della consegna in laboratorio dei campioni fecali ad ogni donatore è stato somministrato un questionario per la raccolta di dati anamnestici rilevanti per le infestazioni da elminti.

Ogni campione di feci è stato insemenzato su 3 piastre di terreno di coltura agarizzato specifico per S. stercoralis (Biolife Italiana s.r.l., Milano), previa omogeneizzazione con acqua distillata se necessario. Le piastre sono state successivamente sigillate con nastro adesivo, per evitare la fuoriuscita di eventuali larve filariformi infettanti, e poste a incubare a $28^{\circ} \mathrm{C}$ fino a 7 giorni.

Quotidianamente è stata eseguita l'osservazione delle colture sia macroscopica (ispezione primaria per verificare la presenza di colonie batteriche cresciute seguendo la scia delle larve), sia microscopica (rovesciando le piastre per ricercare i solchi lasciati dalle larve o le larve stesse).

Al termine dell'incubazione le colture risultate 
negative macro e microscopicamente sono state irrorate con $10 \mathrm{ml}$ formalina al $10 \%$ (forando il coperchio della piastre sigillata con le punte di una pinzetta rese incandescenti alla fiamma) e lasciate a temperatura ambiente per almeno 30 minuti onde devitalizzare le eventuali larve filariformi presenti. Dopo aver delicatamente agitato la piastra, tutta la formalina è stata trasferita in una provetta da $15 \mathrm{ml}$ a fondo conico e centrifugata a 2500 giri $/ \mathrm{min}$ per 5 minuti. Il sedimento è stato osservato microscopicamente per la ricerca di larve e/o vermi adulti di S. stercoralis.

Tutti i campioni sono stati, inoltre, fissati con formalina al 10\% (rapporto 1:3) per almeno mezz'ora e successivamente processati tramite concentrazione fecale con formolo-etil acetato (FEA) per poterne analizzare microscopicamente il sedimento.

Per ogni campione sono stati osservati in tutto 3-4 vetrini (22mm x $22 \mathrm{~mm})$ di sedimento prima dell'annotazione del risultato.

L'esecuzione della concentrazione con FEA è stata effettuata anche per escludere eventuali altre elmintiasi.

\section{RISULTATI}

In tabella 1 sono riportati i livelli percentuali di eosinofilia dei 154 donatori (età media 39.6 aa) sottoposti a "screening" sierologico; il $90 \%$ presentava una eosinofilia $<16 \%$. Sono risultati positivi al test immunoenzimatico 13 donatori, 9 maschi e 4 femmine, pari all' $8.4 \%$ (tabella 2). L'età media dei donatori siero positivi è risultata di 44 anni. In tabella 3 sono riportati i dati riassuntivi dei donatori siero positivi.

Dei 13 donatori positivi allo screening 10 si sono presentati all'approfondimento diagnostico (FEA + coltura). Di questi ultimi 3 (30\%) si sono rivelati positivi alla ricerca colturale per $S$. stercoralis, mentre solo 2 sono risultati positivi con FEA (tabella 4). Complessivamente sono risultati positivi per $S$. stercoralis circa il $2 \%$ dei donatori con elevata eosinofilia (3/154; dati non riportati in tabella). Non sono state evidenziate elmintiasi diverse da strongiloidosi.

In tabella 5 sono indicati i dati riassuntivi degli esami dei sedimenti e colturali eseguiti.

Per quanto concerne i tre donatori risultati positivi, in due casi (Na.M. e Nd.M.) tutti gli esami colturali sono risultati positivi, mentre in un solo campione concentrato con FEA è stato messo in evidenza $S$. stercoralis; nel terzo caso (R.G.) gli esami con FEA sono risultati negativi e un solo esame colturale è risultato positivo.

Questi dati valutati sulla base dei singoli campioni evidenziano la maggior sensibilità dell'esame colturale rispetto all'esame del sedimento fecale.

\section{DISCUSSIONE}

I risultati mostrano che nella nostra provincia, così come in altre parti d'Italia, S. stercoralis è tuttora presente e che può essere trovato anche nella popolazione asintomatica (donatori di sangue).

I dati anamnestici di 9 donatori (in quattro hanno rifiutato di fornire dati) mettono in evidenza uno o più fattori di rischio specifici, soprattutto pratiche agricole o di giardinaggio.

Hanno anche messo in evidenza la scarsa sensibilità dei test di ricerca diretta, in particolare della concentrazione con FEA; sono infatti risultate positive solo il $23.3 \%$ (7/30) delle colture e il $6.7 \%(2 / 30)$ dei sedimenti concentrati.

La scarsa sensibilità della ricerca diretta può essere stata causata dalla caratteristica del parassita di essere, in caso di strongiloidosi cronica, presente in cariche molto basse e dal limitato numero di campioni esaminati per ciascuno dei nostri soggetti.

Per quanto concerne l'età dei soggetti parassitati i nostri dati mostrano come la strongiloidosi possa essere presente anche in giovani adulti, indicando quindi che il parassita persiste sia pur in forma ipoendemica.

In conclusione riteniamo che per escludere una strongiloidosi asintomatica in pazienti destinati ad essere sottoposti a terapie immunodepressive e/o con corticosteroidi, che presentano fattori di rischio quali anamnesi indicativa di predisposizione all'infestazione, ed eosinofilia periferica $>8-9 \%$, sia razionale un approccio diagnostico che preveda in prima istanza la ricerca indiretta immunoenzimatica di anticorpi antiStrongyloides, di semplice esecuzione, e successivamente, in caso di positività, la ricerca diretta per mezzo dell'esame colturale e della concentrazione con FEA da eseguirsi su almeno 6-7 campioni fecali.

Tabella I. Popolazione sottoposta a screening

\begin{tabular}{llll}
\hline Range & Numero & Età & Età \\
\hline eosinofilia (\%) & donatori & media & Range \\
\hline $9.0-15.9$ & 140 & 39.3 & $/$ \\
\hline $16.0-22.9$ & 10 & 40.6 & $/$ \\
\hline $23.0-29.9$ & 4 & 45.5 & $/$ \\
\hline Totale & $\mathbf{1 5 4}$ & $\mathbf{3 9 . 6}$ & $\mathbf{1 8 - 6 4}$ \\
\hline
\end{tabular}

Tabella 2. Screening sierologico

\begin{tabular}{ll}
\hline Totale test sierologici & $\mathrm{I} 54$ \\
\hline Negativi allo screening sierologico & $\mathrm{I} 4 \mathrm{I}$ \\
\hline Positivi allo screening sierologico & $\mathrm{I}$ \\
\hline$\%$ di sieropositività & $8.4 \%$ \\
\hline Età media donatori positivi allo screening & 48 \\
\hline
\end{tabular}


Tabella 3. Dati riassuntivi dei 13 donatori sieropositivi.

\begin{tabular}{|c|c|c|c|c|c|c|c|c|c|}
\hline \multirow[b]{2}{*}{ Donatore } & \multirow[b]{2}{*}{ Sesso } & \multirow[b]{2}{*}{$\begin{array}{l}\text { Paese di } \\
\text { origine }\end{array}$} & \multicolumn{3}{|c|}{ Fattori di rischio } & \multirow[b]{2}{*}{$\begin{array}{l}\text { Contatto } \\
\text { con } \\
\text { animali }\end{array}$} & \multirow[b]{2}{*}{$\begin{array}{c}\text { Eosinofili } \\
\text { per } \mu \mathrm{L}\end{array}$} & \multirow[b]{2}{*}{$\begin{array}{c}\text { Eosinofili } \\
\%\end{array}$} & \multirow[b]{2}{*}{ Assorbanza } \\
\hline & & & Età & $\begin{array}{c}\text { Agricoltura } \\
\text { Giardinaggio }\end{array}$ & $\begin{array}{l}\text { Soggiorno } \\
\text { in Paesi } \\
\text { tropicali }\end{array}$ & & & & \\
\hline G.R. & $\mathrm{F}$ & Italia & 33 & SI & NO & NO & 0.55 & 10.0 & 0.394 \\
\hline M.P. & $F$ & Italia & 29 & I & 1 & 1 & $0.5 \mathrm{I}$ & 10.3 & 0.469 \\
\hline Na.M.* & $M$ & Italia & 59 & SI & $\mathrm{NO}$ & NO & 0.87 & 10.9 & 2.415 \\
\hline M.C. & $\mathrm{F}$ & Italia & 18 & 1 & 1 & 1 & 0.68 & 11.0 & 1.339 \\
\hline C.M. & $M$ & Italia & $4 I$ & $\mathrm{SI}$ & SI & NO & 0.55 & 9.1 & 0.346 \\
\hline T.G. & $M$ & Italia & 54 & $\mathrm{NO}$ & $\mathrm{NO}$ & SI & 0.95 & 15.7 & 0.559 \\
\hline G.T. & $M$ & Italia & 52 & SI & $\mathrm{NO}$ & NO & 0.75 & 13.3 & 0.342 \\
\hline R.G.* & M & Italia & 64 & SI & SI & SI & 0.66 & 10.5 & 0.465 \\
\hline M.G. & $M$ & Italia & 42 & 1 & 1 & 1 & 0.70 & 9.2 & 0.262 \\
\hline P.D. & $M$ & Italia & 31 & $\mathrm{NO}$ & SI & SI & 0.66 & 12.0 & 0.358 \\
\hline Nd.M.* & $\mathrm{F}$ & Senegal & 48 & $\mathrm{NO}$ & SI & NO & 0.58 & 11.8 & 0.476 \\
\hline P.G. & $M$ & Italia & 48 & 1 & 1 & 1 & 0.59 & 9.9 & 0.435 \\
\hline S.S. & $M$ & Italia & 52 & SI & NO & SI & 0.56 & 9.6 & $0.50 \mathrm{I}$ \\
\hline
\end{tabular}

* Donatori che si sono rivelati positivi all'esame coproparassitologico successivo.

Tabella 4. Esami dei sedimenti fecali (FEA) ed esami colturali

Numero donatori positivi allo screening

Numero donatori sottoposti a esame coproparassitologico (FEA + coltura)

Numero donatori positivi all'esame colturale

Numero donatori positivi sia a FEA, sia all'esame colturale

Età media donatori positivi

\begin{tabular}{c}
13 \\
10 \\
\hline 3 \\
2 \\
59
\end{tabular}

Tabella 5. Esami dei sedimenti fecali (FEA) ed esami colturali (dettaglio)

\begin{tabular}{lcccccc}
\hline Donatore & \multicolumn{2}{c}{ Campione I } & \multicolumn{2}{c}{ Campione 2 } & \multicolumn{2}{c}{ Campione 3 } \\
\hline & FEA & Coltura & FEA & Coltura & FEA & Coltura \\
\hline Na.M. & neg & POS & POS & POS & neg & POS \\
\hline C.M.C. & neg & neg & neg & neg & neg & neg \\
\hline T.G. & neg & neg & neg & neg & neg & neg \\
\hline G.T. & neg & neg & neg & neg & neg & neg \\
\hline R.G. & neg & neg & neg & neg & neg & POS \\
\hline M.G. & neg & neg & neg & neg & neg & neg \\
\hline P.D. & neg & neg & neg & neg & neg & neg \\
\hline Nd.M. & neg & POS & neg & POS & POS & POS \\
\hline P.G. & neg & neg & neg & neg & neg & neg \\
\hline S.S. & neg & neg & neg & neg & neg & neg \\
\hline
\end{tabular}

\section{BIBLIOGRAFIA}

1. Abrescia FF, Falda A, Caramaschi G, et al Reemergence of strongyloidiasis, Northern Italy. Emerg Infect Dis. 2009; 15 http://www.cdc.gov/eid/content/15/9/1531.htm.

2. Crotti D, D'Annibale ML, Rossi S. S. stercoralis e strongiloidosi in Umbria: analisi di una casistica decennale. Microbiol Med. 2005; 20: 250-6.

3. Genta R, Gatti S, Linke MJ, Cevini C, Scaglia M. Endemic strongyloidiasis in Northern Italy: Clinical and Immunological Aspects. Quart J Med. 1988; 68 (257): 679-90.

4. Marcos LA, Terashima A, Dupont HL, Gotuzzo E. S. stercoralis hyperinfection syndrome: an emerging global infectious disease. Trans $R$ Soc Trop Med Hyg. 2008; 102: 314-8.
5. Nolan TJ. Canine strongyloidiasis. Companion and exotic animal parasitology. Ithaca: International Veterinary Information Service (www.ivis.org). In: Bowman DD Ed. 2001.

6. Scaglia M, Gatti S, Rondanelli EG. Parassiti e parassitosi umane, dalla clinica al laboratorio. Selecta Medica Ed. 2006; 417-27.

7. Schaffel R, Nucci M, Carvalho E, et al. The value of an immunoenzymatic test (Enzyme-Linked Immunosorbent Assay) for the diagnosis of strongyloidiasis in patients immunosuppressed by hematologic malignancies. Am J Trop Med Hyg. 2001; 65: 346-50.

8. Shoop WL, Michael BF, Eary $\mathrm{CH}$, Haines HW. Transmammary transmission of $S$. stercoralis in dogs. J Parasitol. 2002; 88(3): 536-9.

9. Siddiqui AA, Berk SL. Diagnosis of S. stercoralis infection. Travel Med. 2001; 33: 1041-7. 